\title{
Understanding Complementary Multi-layer Collaborative Heuristics for Adaptive Caching in Heterogeneous Mobile Opportunistic Networks
}

\author{
Vu San Ha Huynh \\ School of Computer Science \\ University of Nottingham \\ Nottingham, United Kingdom \\ vu.huynh@nottingham.ac.uk
}

\author{
Milena Radenkovic \\ School of Computer Science \\ University of Nottingham \\ Nottingham, United Kingdom \\ milena.radenkovic@nottingham.ac.uk
}

\begin{abstract}
Current research aims to deal with emerging challenges of the opportunistic discovery of content stored in remote mobile publishers and the delivery to the subscribers in heterogeneous mobile opportunistic networks. Innovative network and service architectures leverage in-network caching to improve transmission efficiency, reduce delay and handle disconnections. In this paper, we investigate the influences of multi-dimensional heuristics utilised by our adaptive collaborative caching framework CafRepCache on the performance of content dissemination and query in heterogeneous mobile opportunistic environments. We consider the complementary multi-layer heuristics that combine social driven, resources driven, ego network driven and content popularity driven analytics. We extensively evaluate the performance of each complementary heuristic and discuss the impact of each one on every layer of our caching framework across heterogeneous real-world mobility, connectivity traces and use YouTube dataset for different workload and content popularity patterns. We show that the multilayer heuristics enable CafRepCache to be responsive to dynamically changing network topology, congestion avoidance and varying patterns of content publishers/subscribers which balances the trade-off that achieves higher cache hit ratio, delivery success ratios while keeping lower delays and packet loss.
\end{abstract}

Keywords - Opportunistic mobile networks, content discovery and retrieval, content caching, multi-layer heuristics and utilities.

\section{INTRODUCTION}

Large-centralized cloud computing infrastructures provide a range of services and virtually unlimited available resources for users. However, new applications which involve distributed mobile decision making in real time (e.g. real-time HD video streaming, remote heath care, critical applications for public safety communications, augmented/virtual reality apps and automatic driving/traffic control) have emerged with demands which cannot be satisfied by the current approaches due to multiple fundamental challenges [18]. In the scenarios of dynamic mobile disconnection-prone networks, moving from centralised cloud data centre to micro data centres located at the edge of the network [21] is considered as a promising way to deal with the data tsunami and satisfy the ever increasing demand for cloud resources. Current mobile edge/fog computing leverage in-network caching to address complex challenges of improving the reliability of content dissemination and query while reducing the delay and traffic load on the network. In our previous work, we proposed CafRepCache [1], a multi-layer framework that combines real time adaptive multi-path content and interest forwarding, with adaptive cognitive collaborative caching in heterogeneous mobile disconnection-prone environments. CafRepCache addresses complex open questions on end-to-end content retrieval which includes content discovery, content caching and content delivery process, thus aims to maximise cache hit ratio, success ratio while minimizing latency and packet loss.

In this paper, we investigate the influences of different complementary multilayer heuristics and utilities on CafRepCache performance in heterogeneous mobile opportunistic environments. Previous research has shown that collaborative caching usually outperforms locally optimized algorithms [14], thus node utility and content popularity are resolved by both local node and its neighbours. Thus, we address the complex challenge that efficiently utilise local node and ego networks multi-layer real-time predictive heuristics to manage complex dynamic trade-off between dynamically changing topology, mobility, connectivity and content request pattern. Note that we assume there is no malicious behaviour from nodes in the network, thus allow every node in the ego network to collaborate and exchange information and resources with each other as trusted collaborators.

The paper begins by providing an overview of the related work in section II, section III describes collaborative adaptive caching framework CafRepCache and discuss its multilayer heuristics and utilities, section IV evaluates performance of each complement heuristic in content dissemination and query in opportunistic networks across a range of metrics over two heterogeneous realistic mobile social and vehicular traces for real-world workloads and content popularity from YouTube dataset. Section V gives a conclusion.

\section{RELATED WORK}

Current works on content-centric networks typically assume fixed network scenarios [4,5]. With recent advances in wireless mobile communication technologies and devices, 
Mobile Edge Computings (MECs) running as virtual entities have emerged and imposed high degree of mobility of network participants such as subscriber mobility [4]. Mobile publisher data problem is still not understood sufficiently clearly [6] and leads to lower quality of the services. Authors in $[15,16,17]$ considered caching content at the edge of the mobile networks as the promising approach to address the network traffic demands from mobile users in which public or private transportation may act as mobile relays and caches. In our previous work [18], we envisaged a low cost mobile personal cloud architecture that can host multiple different services which are distributed, dynamic, operate in real time, adaptive to environment and uses intelligent network communication for reliability.

Authors in [10] combine betweenness, similarity and tie strength for social routing metric which forwards content packets to node that have higher social degree in order to increases the probability of delivering packets to the destination. However, overusing a high central node will make it congested, results in the congestion in part of the network or even the whole network. Furthermore, overload in a node may cause extremely quick battery drainage and make the node totally unusable. [2] proposed Cafe and CafRep, a congestionaware mobile social framework for data forwarding and replication over heterogeneous opportunistic networks. Cafe and CafRep maximise message delivery ratio and availability of nodes while minimising latency and packet loss rates by detecting and avoiding congestion on a node or part of nodes. In [3], authors proposed fully distributed mechanism for next generation mobile offloading in resource-constrained mobile networks that delegates traffic to other nodes based on its remaining resources, contact frequency and duration. Authors in [13] studied the effect of content popularity ranking in cooperative caching schemes on delay tolerant mobile network and designed a mathematical model of mobile content sharing network based on file popularity distribution, user mobility and delay tolerance. Our previous work [1] described CafRepCache as our early proof of concept about new latency aware collaborative cognitive caching approach in heterogeneous opportunistic mobile networks which utilises fully localised and ego networks multi-layer predictive heuristics about dynamically changing topology, resources and popularity content. In this paper, we discuss and investigate the impact of each CafRepCache's complementary multilayer heuristics on the performance of end to end content retrieval in mobile heterogeneous opportunistic environments.

\section{ADAPTIVE COLLABORATIVE CACHING FRAMEWORK}

In this section, we describe our adaptive collaborative caching framework architecture CafRepCache, design space and discuss its complementary multi-layer predictive heuristics and utilities about dynamically changing topology, resources and content popularity.

\section{A. Framework Architecture}

Our adaptive collaborative caching framework CafRepCache has a distributed cross-layer structure [1] and multi-dimensional design space as shown in Fig. 1. Our work aims to improve the reliability and scalability of current state-

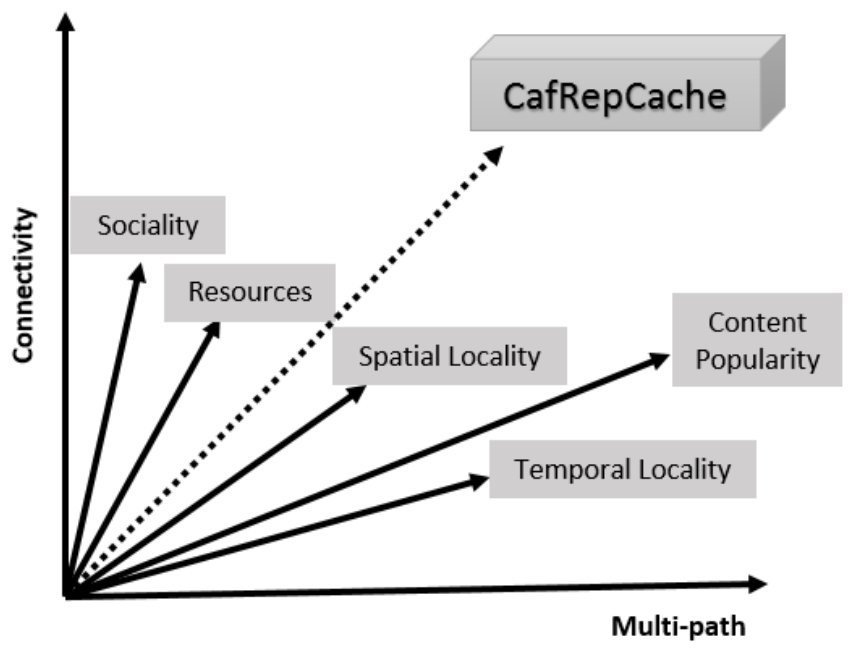

Figure 1. Complementary multi-dimensional collaborative heuristics

of-the-art content dissemination and query in mobile disconnection-prone networks. In order to achieve the ultimate goal that maximize the cache hit rate and content delivery success ratio as well as reduce latency and packet loss, we address multi-dimensional issues related to content/interest routing and forwarding, content searching and caching decisions (e.g. which data to cache, where to cache, and how to manage the cache). Our multi-layer approach is a key to enabling the resources-and-latency-aware content forwarding, real-time predictive content caching that support varying workloads, dynamically changing content popularity, dynamic mobility and connectivity in both dense and sparse environments.

Our design combines: i) social driven heuristics that aim to improve the content delivery success ratio and cache hit rate by selecting the intermediaries or caching points with higher social degree for forwarding and caching contents; ii) node resources driven heuristics that aims to detect and avoid overload of the nodes that have high social centrality but low buffer availability, high delays or high congesting rate; iii) ego network driven heuristics that aims to not only consider local observations but also value collaborative observations from nodes within its ego network. Note that we define ego network as a dynamic network consisting of the node itself and contacts it meets most frequently or recently. Ego network allows each node to give its own spatial or temporal perspective of the network. Ego network applied to resourcesaware heuristics helps to detect and avoid parts of the networks that have low buffer and increased delay, then offload the traffic from more to less congested parts of the network; iv) content driven heuristics that aim to take advantage of highly skewed content popularity and content request spatial and temporal locality to efficiently predict the incoming content requests. We first describe in detail our multi-layer complementary analytics, heuristics and utilities in the below section, then evaluate the influences of each heuristic and utility on the performance of content dissemination and query. 


\section{B. Complementaty Multi-layer Collaborative Heuristics}

a) Social Forwarding Consideration: Social utility is collaboratively analysed and resolved based on complex network metrics [1,10]: similarity, betweenness and tie strength that is used to direct the traffic to higher centrality nodes for caching contents or forwarding requests in order to increase the chance that requested contents are delivered successfully to subscribers (i.e. the success ratio). Social utility of node $n_{i}$ is measured by the Betweenness value of $n_{i}$ as well as the Similarity and Tie Strength between $n_{i}$ and subscriber s of content k at time t: $s \in S_{k}^{t}$.

- Betweeness:

$U S_{i, s}^{B e t}=\sum_{a=1}^{N} \sum_{b=1}^{a-1} \frac{g_{a b}\left(n_{i}\right)}{g_{a b}}, g_{a b}\left(n_{i}\right)$ is the number of paths linking node $a$ and node $b$ that includes $n_{i}$.

- Similarity:

$U S_{i, s}^{S i m}=\left|N_{i} \cap N_{s}\right|$ is the similarity of contact between node $n_{i}$ and subscriber $s$.

- Tie Strength:

$U S_{i, s}^{T S}=\frac{f(s)}{F(n)-f(s)}+\frac{\operatorname{rec}(s)}{T(n)-\operatorname{rec}(s)}+\frac{d(s)}{D(n)-d(s)} \quad$ combines both frequency and recency of contacts between cache node $n_{i}$ and subscriber $s$.

- Ego Network Social Utility:

$$
U S_{i, s}^{E N}=\frac{1}{E N} \sum_{n_{j} \neq n_{i} \in E N} U S_{j, s}^{B e t}+U S_{j, s}^{S i m}+U S_{j, s}^{T S} \text { is the }
$$
social utility value of $n_{i}$ 's ego networks.

Our social heuristics utilise SimBetTS Routing metric [10] as it comprises of both node's social degree and others' social centrality within its ego network. Our work is not intended to be limited to using only SimBetTS metric but could be integrated with other social metrics as well.

SocialUtil $_{i, s}=\frac{1}{|F|} * \sum_{f \in F} \alpha U S_{i, s}^{f}$

$f \in F=\left\{\right.$ Social, $E N_{\text {Soc }} \mid$ Social $=$

\{Betweeness, Similarity, TieStrength\}\}

b) Node Resource Consideration: Our node-resource and delay driven analytics include retentiveness - defined as percentage of remaining storage capacity in a node, receptiveness - defined as predictive in-network delay observed by the node, congesting rate - defined as indication of how fast the buffer is to get filled up, as below.

- Retentiveness:

$U R_{i}^{R e t}=\theta_{i}-\sum_{o_{k} \in O} \delta_{k}$ as the node's available storage at time $t$, measured by sum of all cached content occupancy subtracted from the node's cache buffer capacity.

- Receptiveness:

$U R_{i}^{\text {Rep }}=\sum_{o_{k} \in O} t_{\text {now }}-t_{\text {received }}^{i}$ as the delay node $n_{i}$ adds to content $o_{k}$, measured by the sum of differences between the current time and the time each content was received.

- Congesting Rate:

$U R_{i}^{C R}=\left(\frac{t_{\text {end }}^{\text {congestion }}-t_{\text {start }}^{\text {congestion }}}{t_{\text {start }}^{\text {congestion }+1}-t_{\text {end }}^{\text {congestion }}}\right) \frac{f}{e^{f}} \quad$ where $\quad t_{\text {start }}^{\text {congestion }}$ or $t_{\text {end }}^{\text {congestion }}$ denotes the time when congestion occurs or finishes, $t_{\text {start }}^{\text {congestion }}$ and $t_{\text {start }}^{\text {congestion }+1}$ are two adjacent congestions occurred, $f$ is the observed congestion frequency. For simplicity, we assume $f=1$.

- Ego Network Resource Utility:

$U R_{i}^{E N}=\frac{1}{E N} \sum_{n_{j} \neq n_{i} \in E N} U R_{j}^{R e t}+U R_{j}^{R e p}+U R_{j}^{C R} \quad$ is the resource utility value of $n_{i}$ 's ego networks

$$
\text { ResourceUtil }_{i}=\frac{1}{|F|} * \sum_{f \in F} \alpha U R_{i}^{f}
$$

$f \in F=\left\{\right.$ Resource, $E N_{\text {Res }} \mid$ Resource $=$

\{Retentiveness, Receptiveness, CongestingRate\}\}

c) Content Popularity Consideration: Content utility is resolved dynamically by performing caching decisions on whether to cache, forward or delegate content chunks based on local and collaborative content popularity observations. Intuitively, node $n_{i}$ 's content utility will be maximised by caching the highest popularity content, thus improves the cache hit rate. Moreover, node $n_{i}$ will also gain content utility by helping to redirect the request to a collaborative cache and a hit is attained there. The utility gained is proportional to the size of the cached content. Our content popularity analytics is defined as:

$$
P(\mathrm{Ti})=\frac{\text { ObservedTimePeriod }(\mathrm{Ti}) *}{\text { Total }- \text { time } * \text { Betweeness }(\mathrm{Ti}) * \text { Recency }(\mathrm{Ti})}
$$

$P(T i)$ measures probability caching decision over a certain period (i.e. temporal locality) in which $P$ is the weight that identify the content popularity. Betweeness(Ti) is the temporal function that measures the time gap between continuous requests and Recency(Ti) denotes the most recent interest request. $\mathrm{P}(\mathrm{Ti})$ aims to provide tradeoff between current observed content popularity versus long terms interest in it in order to balance between potentially fake news and long term useful content. When a caching node detects it is likely to start congesting, it ranks the content in terms of its popularity and delegates the least popular content to a suitable node. Nodes suitability is ranked in terms of the same multi-criteria metric we described (social, resources and workload).

\section{EVALUATION}

In this section, we present and evaluate the influences of different heuristics and utilities on the performance of content dissemination and query protocols while varying the patterns of subscribers requesting contents from a fixed number of publishers. As mobility and connectivity of the nodes have a major impact on the performance of any opportunistic communication protocol, it is fundamental to evaluate our caching algorithm over multiple heterogeneous real-world mobile data sets. We use San Francisco Cab [11] and Infocom [12] traces in ONE [9]. San Francisco Cab Trace [11] includes GPS traces of 550 cabs over a period of 30 days in the San Francisco Bay Area. Infocom [12] is a 4-day trace that consists of 78 volunteers equipped with Bluetooth devices and additional 20 static long-range devices placed at various semistatic and static locations of the conference venue. San Francisco trace [11] is the more challenging trace compared to Infocom [12] due to very short connectivity durations, very high disconnections and low number of contacts during connected times. 
For content, we use YouTube Dataset $[7,8]$ as a real trace for content requests. The dataset contains total 2135798 videos, each has video id, length, views and other attributes. Based on the assumption that file size is proportional to the video length, we set up the average file size is $8.4 \mathrm{MB}$, which is in line with $[7,8]$. We also assume that contents are uniformly distributed among publishers. All caching points in the network have a uniform storage capacity.

In the first set of experiments, we evaluate and compare five different content forwarding protocols in opportunistic networks including Random Forwarding, Epidemic Forwarding, Social Local Forwarding, Social Ego Network (EN) Forwarding and Social Local+EN Forwarding. Random Forwarding protocol forwards contents and interest packets to a random encountered node. Epidemic Forwarding protocol floods the contents and interest packets to all nearby contacts. Social Local Forwarding is a forwarding protocol based on sociality metrics observed by the node itself while Social EN Forwarding is based on sociality degree measured by other nodes within its ego network. Social Local+EN Forwarding consists of social utilities observed from both local node and its ego network. We apply simple LRU caching algorithm built on top of content forwarding protocols and evaluate the performance of content dissemination and query over different criteria: success ratio, delay and packet loss in the presence of dynamically workload, dynamic mobility pattern and connectivity. Due to space limitation, only the success ratio and delay will be shown.

In the second set of experiments, we conduct performance analysis of five different content forwarding protocols built with our resources awareness algorithm [2] while dynamically varying the number of subscribers in order to evaluate the influences of our resources aware heuristics on the performance of content dissemination and query in terms of cache hit ratio, success ratio, delay, packet loss and relative footprint reduction.

In the third set of experiments, we evaluate the performance of five forwarding protocols with resource awareness and our cognitive collaborative caching algorithms built on the content layer [1] in order to analyse the effect of our adaptive content-aware caching protocol on content dissemination and query performance in mobile disconnection prone environments in the presence of dynamic content request patterns, dynamic mobility and connectivity.

\section{A. Evaluation of forwarding heuristics and utilities}

We run six increments with percentage of random subscribers ranging from $10 \%$ to $60 \%$ as we observe that our content dissemination and query performance converges when the percentage of random subscribers gets larger than $>60 \%$. For each experiment, we assume that a random $25 \%$ of nodes are publishers as in line with [1,2]. Subscribers and publishers are mobile and can have different connectivity patterns. We also assume that contents are uniformly distributed among publishers. For simplicity and to provide sensitivity analysis of content size, we limit the number of content to 100000 without losing generality. We assume that all caching points in the network have a uniform storage capacity such that the total caching space could fit at $0.1 \%$ of the total contents population. All experiments are repeated ten times and averaged.

We begin by analysing performance of end-to-end success ratio and delay for content retrieval. In Fig. $2 \mathrm{a}$ and $3 \mathrm{a}$, we show that the success ratio of all forwarding protocols decreases whilst the delay raises up in the face of increasing content workload and level of congestions. SocialLocal+EN Forwarding achieves the highest success ratio and lowest average delay (around 40\% success ratio, $135 \mathrm{~min}$ delay for SF traces and $60 \%$ success ratio, 10 min delay for Infocom traces) whilst Random Forwarding gets the lowest success ratio and highest delay (around 20\% success ratio for both traces, $167 \mathrm{~min}$ and $18 \mathrm{~min}$ delay for SF and Infocom traces respectively) compared to other forwarding algorithms Epidemic Forwarding, SocialLocal Forwarding and SocialEN Forwarding. This is because SocialLocal+EN Forwarding profits from adaptive collaborative social utility resolved based on complex network metrics from both its own observation and other nodes' observations in ego network in order to find the higher centrality nodes for forwarding requests that increases the chance that requested contents are delivered successfully to subscribers. SocialLocal+EN Forwarding performance is followed by SocialLocal Forwarding and SocialEN Forwarding which manages up to
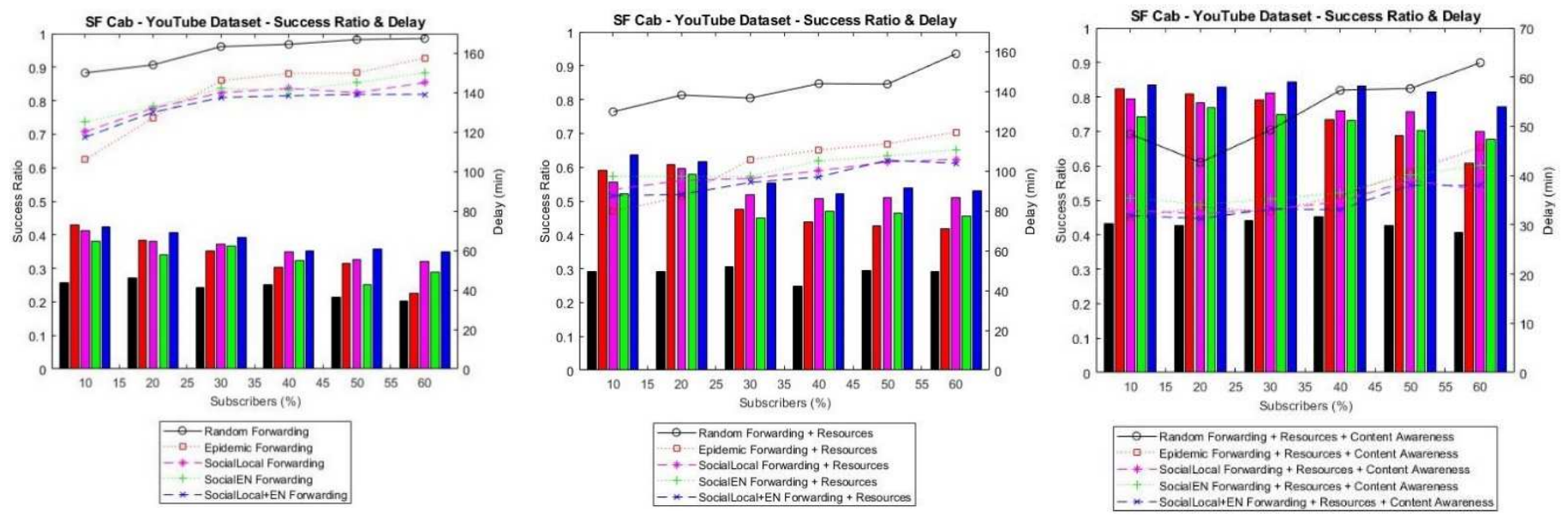

Figure 2. $a, b$, c. San Francisco Cab data trace success ratio and delay vs. Number of subscribers 

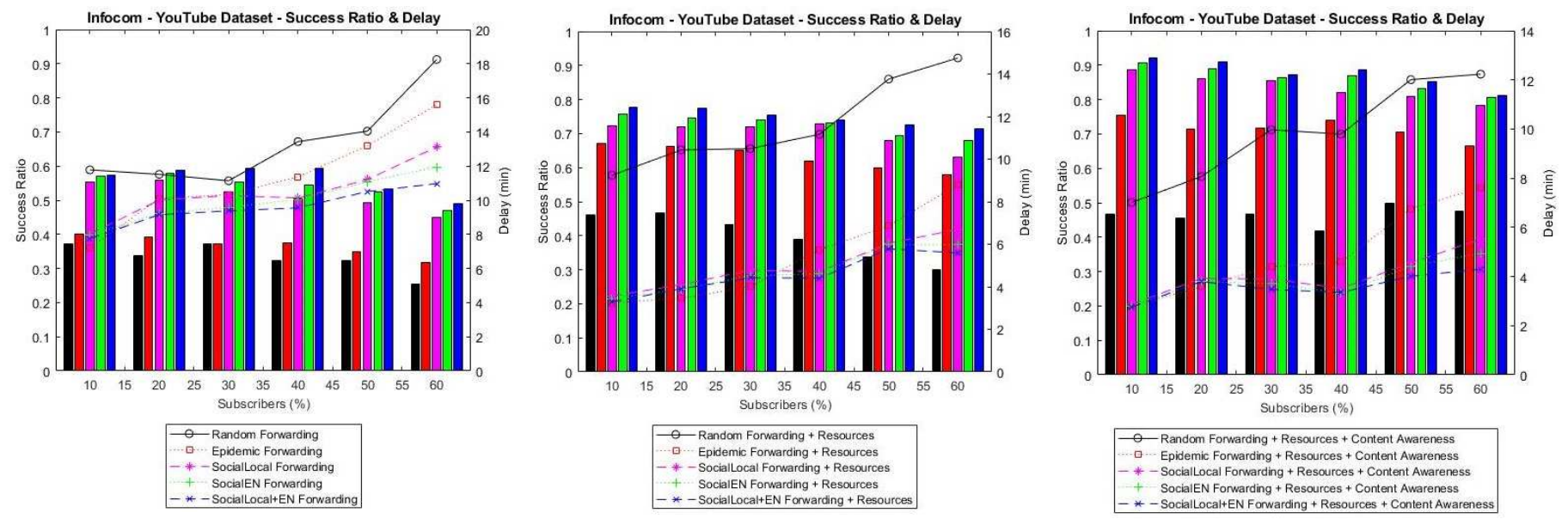

Figure 3. $a, b$, c. Infocom data trace success ratio and delay vs. Number of subscribers

$35 \%$ success ratio for SF traces and $52 \%$ for Infocom traces, delay ranges from above $148 \mathrm{~min}$ for $\mathrm{SF}$ traces and $12 \mathrm{~min}$ for Infocom traces. We argue that social utility resolved by either only local observation (as in SocialLocal Forwarding ) or egonetwork observation (as in SocialEN Forwarding) is not sufficiently adaptive to the dynamic node's mobility and connectivity. Moreover, it is interest to see that ego network plays less important role than local node in San Francisco Cab trace compared to Infocom traces such that SocialLocal Forwarding achieves higher success ratio and lower delay than SocialEN Forwarding for the SF Cabs trace (and vice versa for Infocom 2006 traces). This is due to SF Cabs having the least repeating mobility patterns and thus not benefiting significantly from the nodes' ego network predictions for future forwarding. So the most greedy approach of SocialLocal Forwarding works better for SF Cab trace while its performance is worse than SocialEN Forwarding for the more social Infocom traces. Epidemic Forwarding starts off with relatively good success ratio and delay $(40 \%$ success ratio for both traces, 117 min delay for SF traces and 9 min for Infocom traces) for low to medium percentage of subscribers but then decreases to about $20 \%-29 \%$ success ratio and increases the delay to about $160 \mathrm{~min}$ for SF traces and $15 \mathrm{~min}$ for Infocom traces in the face of increasing congestion and workload.

\section{B. Evaluation of resources-aware heuristics and utilities}

In the previous sub-section, we show that all forwarding protocols performance decrease in the presence of increasing content workload and level of congestions. This is due to either flooding scheme will greatly waste network and bandwidth resources or directing traffic to the higher central nodes will eventually congest that nodes. In this section, we evaluate performance analysis of the five content forwarding protocols built with our resources awareness algorithm [1,2] while dynamically varying the number of subscribers in order to measure the influences of resources aware heuristics and utility on content dissemination and query performance in terms of cache hit ratio, success ratio, delay and packet loss. Due to space limitation, only success ratio and delay will be shown.
In Fig. $2 b$ and $3 b$, we show that resources awareness heuristics and utility improve the success ratio and delay of all five forwarding protocols compared to that without resources awareness in the face of increasing congestion level. When the number of subscribers increases, SocialLocal+EN Forwarding + Resources still keeps above $57 \%$ success ratio for SF traces and $75 \%$ for Infocom traces, up to 100 min delay for SF traces and 6 min for Infocom traces. SocialLocal+EN Forwarding + Resources is more successful compared to SocialLocal+EN Forwarding without resource awareness as it performs innetwork predictive resource analytics and rebalances the network traffic so that it detects and avoid congested nodes and congested parts of the network by using implicit hybrid contact and resources congestion heuristics while keeping high social metrics to drive content to more central nodes that increase the possibility to deliver content to the subscribers. Our resource-aware mechanism [1,2] exploits localised relative utility to offload the traffic from more to less congested parts of the network, and to replicate adaptively in different parts of the network with non-uniform congestion levels. SocialLocal+EN Forwarding + Resources is followed by SocialLocal Forwarding + Resources and SocialEN Forwarding + Resources which achieves up to $60-70 \%$ success ratio for both traces, above 110 min delay for SF trace and $8 \mathrm{~min}$ for Infocom. Epidemic Forwarding + Resources shows the improvement in success ratio and delay due to its resource-controlled flooding although it still suffer from the scalability issues in the increase of number of subscribers which achieves up to $60 \%$ success ratio for both traces, above 118 min delay for SF traces and 10 min for Infocom traces. Random Forwarding + Resources gets the least benefit from the resources awareness as it does not utilise efficiently the network resources but only forward the contents and interest packets to random node, thus achieve the lowest success ratio and highest delay for both traces.

\section{Evaluation of content-aware caching heuristics and utilities}

In the previous subsections, we apply simple LRU caching algorithm for content dissemination and query. In this section, we conduct performance analysis of the influences of our 
cognitive collaborative caching algorithms [1] built on top of the five forwarding protocols with resources awareness.

In Fig $2 \mathrm{c}$ and $3 \mathrm{c}$, we show that SocialLocalEN Forwarding + Resources and Content Awareness which is our adaptive collaborative caching framework CafRepCache [1] achieves the best performance in term of success ratio and delay. It manages to keep above $80 \%$ success ratio for both traces, below 40 mins delay for SF traces and 5 mins for Infocom traces in the face of increasing workload and congestion levels. CafRepCache is most successful as it includes both social and resource metrics, and profits from adaptive caching as it takes advantage of highly skewed content popularity and content request locality to efficiently predict the incoming content requests, thus manages to select highly suitable contents and locations for caching. Moreover, SocialLocalEN Forwarding + Resources and Content Awareness protocol (or CafRepCache) outperforms others also because CafRepCache is able to delegate caching of the least popular content from its local cache to another node with higher resources and that still has the highest chance to get a cache hit amongst all the contacts it meets.

\section{CONCLUSION}

This paper focuses on understanding the influences of fully localised and ego networks multi-layer predictive heuristics on the performance of collaborative adaptive caching in real world dynamically changing mobile topologies. Our results show that multi-path social content and interest forwarding with resources awareness and adaptive collaborative content caching helps to improve significantly the performance of content dissemination and query in complex temporal fragmented mobile environments. We show that CafRepCache utilises fully localised and ego networks social, resources and content heuristics about dynamically changing topology, resources and content popularity, thus achieves high success ratio and caching efficiency, low delays and packet loss.

For our future work, we plan to investigate the energy efficient data sharing approaches in CafRepCache in order to decrease the operating costs, increase reliability and scalability in opportunistic disconnection tolerant networks. We also plan to investigate a reputation system $[19,20]$ integrated with CafRepCache to deal with malicious behaviour, incentivise nodes to collaborate, increase security and privacy. We plan to build and deploy a real-world testbed which integrates CafRepCache with a lightweight publish/subscribe messaging transport protocol designed for connecting constrained devices, people, vehicles and infrastructure to contribute towards integrative smart city planning.

\section{REFERENCES}

[1] Radenkovic, Milena and Huynh, Vu San Ha. Collaborative cognitive content dissemination and query in heterogeneous mobile opportunistic networks. SMARTOBJECTS 2017 (3rd Workshop on Experiences with the Design and Implementation of Smart Objects), Mobicom USA 2017.
[2] M. Radenkovic, A. Grundy. Efficient and Adaptive Congestion Control for Heterogeneous Delay Tolerant Networks. Ad Hoc Networks, v. 10, n. 7, 2012.

[3] H. Flores, R. Sharma, D. Ferreira, V. Kostakos, J. Manner, S. Tarkoma, P. Hui, Yong L. Social-aware hybrid mobile offloading. Pervasive and Mobile Computing 36, p.25-43, 2017.

[4] D. Grewe, M. Wagner, M. Arumaithurai, I. Psaras, and D. Kutscher. Information-Centric Mobile Edge Computing for Connected Vehicle Environments: Challenges and Research Directions. Proceedings of the Workshop on Mobile Edge Communications (MECOMM '17). ACM, USA. 2017.

[5] C. Fang, F. R. Yu, T. Huang, J. Liu, and J. Liu. A survey of green information-centric networking: Research issues and challenges. IEEE Commun. Surveys Tutorials, 2015.

[6] J. Augé, G. Carofiglio, G. Grassi, L. Muscariello, G. Pau, and X. Zeng. MAPMe: Managing Anchor-less Producer Mobility in InformationCentric Networks. CoRR abs/1611.06785 (2016).

[7] M. Cha, H. Kwak, P. Rodriguez, Y.-Y. Ahn, and S. Moon. I tube, you tube, everybody tubes: Analyzing the world's largest user generated content video system. Proc. ACM IMC, Oct. 2007.

[8] Xu Cheng, C. Dale, and Jiangchuan Liu. Statistics and social network of youtube videos. 16th Int. Workshop on Quality of Service (IWQoS), p. 229-238. 2008.

[9] A. Keränen, J. Ott and T. Kärkkäinen. The ONE Simulator for DTN Protocol Evaluation. SIMUTools'09: 2nd International Conference on Simulation Tools and Techniques. Rome. 2009.

[10] E. Daly and M. Haahr. Social network analysis for routing in disconnected delay-tolerant manets. Proceedings of ACM MobiHoc, 2007

[11] San Francisco cabs data traces, downloaded from the Cab Spotting project, $<$ http://cabspotting.org/api $>$ for the period 20 Sep 2011- 20 Oct 2011

[12] Scott, R. Gass, J. Crowcroft, P. Hui, C. Diot, A. Chaintreau. CRAWDAD data set Cambridge/haggle (v. 2009-05-29), May 2009.

[13] T. Wang, P. Hui, S. R. Kulkarni, and P. Cuff. Cooperative Caching based on File Popularity Ranking in Delay Tolerant Networks. Proc. ExtremeCom, Zurich, " Switzerland, March 2012.

[14] D. Bertsimas, V. F. Farias and N. Trichakis. The price of fairness. Operations Research. v. 59, n. 1, 2011.

[15] L. Vigneri, T. Spyropoulos, C. Barakat. Storage on Wheels: Offloading Popular Contents through a Vehicular Cloud. IEEE 17th International Symposium on A World of Wireless, Mobile and Multimedia Networks (WoWMoM) 2016.

[16] L. Vigneri, T. Spyropoulos, C Barakat. Streaming Content from a Vehicular Cloud. Proceedings of the Eleventh ACM Workshop on Challenged Networks (CHANTS '16). ACM, New York, NY, USA, p. 39-44. 2016.

[17] L. Vigneri, S Pecoraro, T. Spyropoulos, C. Barakat. Per-Chunk Caching for Video Streaming from a Vehicular Cloud. Proceeding CHANTS '17 Proceedings of the 12th Workshop on Challenged Networks p.57-62. USA 2017.

[18] Milena Radenkovic. Cognitive Privacy for Personal Clouds. Cognition and Cooperation in Advanced Wireless and Mobile Information Systems, Mobile Information Systems. 2016(1): 31. 2016

[19] Sonja Buchegger. Reputation Systems for Self-Organized Networks: Lessons Learned. IEEE Technology and Society Magazine, Toward Fourth Generation Wireless, p. 1-10. 2008.

[20] Sonja Buchegger, Jochen Mundinger, Jean-Yves Le Boudec. Reputation Systems for Self-Organized Networks. IEEE Technol. Soc. Mag. 27(1) p. 41-47. 2008

[21] Milena Radenkovic and Natasa Milic-Frayling. 2015. Demo: RasPiPCloud: A Light-weight Mobile Personal Cloud. In Proceedings of the 10th ACM MobiCom Workshop on Challenged Networks (CHANTS '15). ACM, New York, NY, USA 